\title{
Quantifying Aesthetic Parameters of Web Applications
}

\author{
Raj Kulkarni, Ph.D. \\ Walchand Institute of Technology \\ Solapur University, \\ Solapur MH India
}

\author{
P.S.R. Patnaik \\ Walchand Institute of Technology \\ Solapur University, \\ Solapur MH India.
}

\begin{abstract}
:
Web applications and web sites are integral part of day to day life, most of the activities like searching, banking, shopping, paying utility bills and other activities are carried online through web. Visual aspects and its aesthetic appeal of any web application are crucial factors to determine the success of web applications. In this paper we study aesthetics factors which form visual appeal of a web application to retain its visitors. And develop a tool based on VIPS algorithm to compute the aesthetic factors of web applications. This study considers prominent factors like layout, economy, unity, font size, color, proportion, harmony, and variety to calculate the aesthetic factors computationally. Earlier evaluations methods for aesthetic factors are either manual or survey based. This work attempted to compute the aesthetic factors and score automatically using own developed tool.
\end{abstract}

Keywords: Web Applications, VIPS, Aesthetic Parameters.

\section{INTRODUCTION}

Aesthetics is the factor which decides the popularity of the web application. Aesthetics also provides a way to decide how the site will fare in the long run as the predictions of web applications are in done in 30 seconds. It is conclusive that although the criteria by which people judge visual appearance of, user satisfaction and trustworthiness are not defined, visual appeal which relates to aesthetics is still a subject of study. It is to be noted that information and search sites would also attempt to communicate with good visuals. Icons, images are an useful way to communicate and have more attention grabbing effects on the users.

David Siegel, who in the book" Creating Killer Web Sites" from 1996 argued for the importance of aesthetic dimensions, was a proponent of aesthetic Web sites. On the other hand The critics argue it does not matter whether the website was blue or red as long as it was functional and user-friendly.

Aesthetics play a important role in creating the Image of the organization or the person behind the web application. It is therefore very imperative that even Web Applications that mostly have an information purpose also aesthetically reflect the organization behind the site.

We have reached an era where the technical and functional aspects of Web applications are now defined and finalized .It is now perceived that the web applications just work perfectly without any flaws. The technology is viewed as a basic foundation for aesthetic experiences.

Aesthetic is the one of the crucial deciding factor for retention of visitors on website. There have been several attempts to define and quantify the aesthetics parameters of a website. The six aesthetics related elements used are balance, equilibrium, symmetry, sequence, rhythm, as well as order and complexity. We discuss some of aesthetics parameters relevant to websites and measure them using a VIPS algorithm. In our novel attempt to quantify the aesthetic aspects of website we see very promise able results.

\section{RELATED WORK}

There has been lot of work carried out in study of aesthetics and its effects on software user interfaces. Most of the research was heuristics based. The previous research mostly focused on those factors of aesthetics which are not much pertaining to websites. $\mathrm{Ngo}$ et al comprehensively defined the aesthetics factors and also formalized various formulas which could quantify the aesthetics aspects of software interface and also empirically attempted to verify the accurateness and correctness of the computational theory of evaluating interface aesthetics. Carolyn Salimun[2] et al worked on six layout principles (Cohesion, Economy, Regularity, Sequence, Symmetry, and Unity).They found that the layout principles of Symmetry and Cohesion are more influential than the other layout principles. Similar work to quantify the aesthetic aspects was made in study of artistic aspect of software and web interfaces.

A study was conducted by Parizotto-Rebeiro and Hammond [3] which investigated several Gestalt principles like unity, homogeneity, proportion, balance, and rhythm. In that results compared the effect of the extent of aesthetic presence in each layout principle (. good unity vs bad unity), but the comparison did not take in consideration the effect between principles (good unity vs. good balance)

Lisbeth Thorlacius in its article emphasizes on the role of aesthetics in web design [5] discusses the four main areas in which Aesthetics play an important role in the design of successful Web sites: aesthetics play an important role in supporting the content and the functionality, in appealing to the taste of the target audience, in creating the desired image for the sender, and in addressing the requirements of the Web site genre. The author describes his views on the balance of functionality and aesthetics, argues that visual communication through aesthetics is vital for content perception to users and better aesthetic appeal of the website. Hence its quite important to make aesthetic features of website computable.

A tool called BWAIN [6] was developed to artistically interpret a website correlating website structure with sound and visual art.

Visual aesthetics have been shown to enhance positive user experience in the context of the World Wide Web (WWW) Aesthetics is now center point factor in analysis of web applications. The very first impressions of viewing a web site's home page affect a user's decision as whether to continue viewing that web site or move to another one. This decision can be made in as little as 50 milliseconds mainly because of the page's visual appeal [10]. First impressions affect users' judgments on different 
aspects of web site design including usability, credibility or purchasing intentions [11].

Recent work by HP Inc fitted Gaussian Model to aesthetic parameters of document layout [12]

\section{VISION BASED PAGE SEGMENTATION (VIPS)}

The VIPS algorithm [4] has been developed to extract the semantic structure for a web page. Segmentation detail is controlled through the granularity parameter of VIPS, which controls the coherence of identified segments based on visual perception. By using maximum granularity value, this process aims to identify the smallest possible visual segments, which will be concatenated through simple heuristics to form the basic building blocks, such as headers, footers, columns, headings, paragraphs, tables, images and captions. Such semantic structure is a hierarchical DOM like structure in which each node will correspond to a block. Degree of Coherence value is assigned to each extracted block to indicate how coherent of the content in the block based on visual perception.

The VIPS algorithm makes full use of page layout feature: it first extracts all the suitable blocks from the html DOM tree, then it tries to find the separators between these extracted blocks. Separators are typically the horizontal or vertical lines in a web page that visually cross with no blocks or color groupings. Finally, based on these separators, the semantic structure for the web page based on its layout, coherence is constructed. Top down approach and Degree of Coherence of VIPS algorithm makes it is very effective for analyzing aesthetic features of web page.

Degree of Coherence (DoC) is a measure denoting how coherent it is. DoC has the following properties:

1) The greater the DoC value, the more consistent the content within the block;

2) In the hierarchy tree, the DoC of the child is not smaller than that of its parent.

In VIPS algorithm, DoC values are integers ranging from 1 to 10 , moreover different ranges. Permitted Degree of Coherence (PDoC) is used to achieve different granularities of content structure for different applications. The smaller the PDoC is, the coarser the content structure would be. Different application can use VIPS to segment web page to a different granularity with proper PDoC. Different Selection of PDoC values can effectively be used to analyze aesthetic features [9] namely layout, economy unity, harmony, variety of web page.

The research reported here investigated the relationship between user preferences of the aesthetic design of interfaces, based on six of the aesthetics formulae defined by Ngo et. al. [7]]

\section{AESTHETIC FEATURES FOR WEB APPLICATIONS}

Layout balance: This measures the spatial distribution of visual element in a webpage. If an element is deemed balanced, viewers feel a greater sense of stability, which in turn contributes to feelings of cohesion and equilibrium. Visual elements seem to have a "weight" which depends on distance from a central point and the relative distance to other elements which VIPS handles effectively.

Color Balance: Conveys the average spatial distribution of colors used across data visualization. Instead of determining whether pixels are belonging to the foreground or the background, Color Balance represents how colors are distributed over the visual element.
Economy: Metric represents the efficiency of the visual artifact created by the data visualization technique to convey a specific dataset. In general, aesthetic objects are streamlined, using no extraneous elements and presented with optimum efficiency [7]. The notion of consistency through economy as an aesthetic principle is supported by the requirement that attractive displays should "have a properly chosen format and design" [8]. Economic images contain elements with matching colors and similar sizes, and so on. If a display is economic, a user feels focused through the visual simplicity of the message; each single element displayed has a distinct purpose.

Unity: It is considered as a spatial distribution measurement of elements. Unity [9] thus conveys how the visualization is perceived as "a whole" rather than a collection of separate parts. Unity is then determined by the amount of "empty" or "unused" space between graphical elements: the greater this space, the more separate elements stand out, and the less unified the visualization is.

Harmony: It is determined by the comparative relationship between dimensions of elements inside the visualization relatively to each other, and to defined ,aesthetic proportions" [7]. Displays that reflect a sense of harmonious proportion are deemed as more attractive [8]. Two different categories of aesthetic proportion aspects are considered, distinguished whether the visualization is perceived as an individual form, or as a coherent constellation of several similar elements.

Variety: Implies the nature and extent of novelty which the visual elements communicate to user. It reflects low predictability in that a visual element has to be highly informative and rich in content if the content cannot be easily deduced. Variety as a aesthetic feature to be optimal is equivalent to high complexity or low redundancy, is achieved by using a high amount of different elements and distributing these elements equally in the webpage or image in order to maximize the unpredictability of elements occurring.

\section{METHODOLOGY OF QUANTIFYING THE AESTHETIC FEATURES OF WEB APPLICATION}

\subsection{Symmetry}

VIPS algorithm obtains the blocks by segmenting the page visually. We see the balance of subtree obtained as a measure of symmetry.

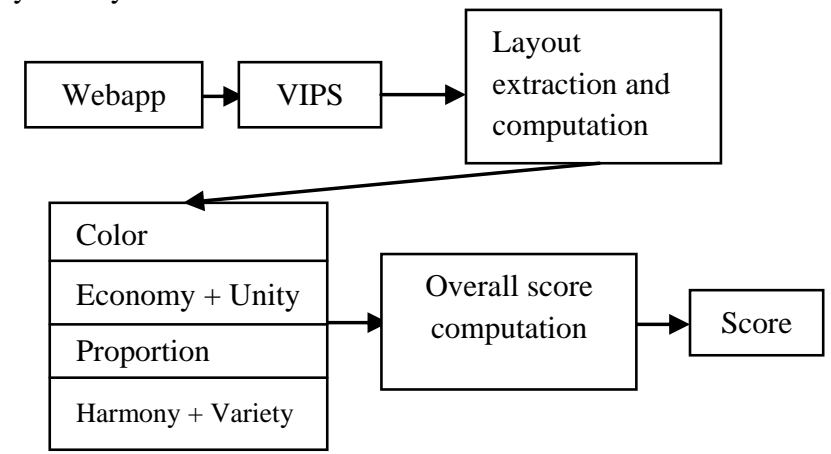

Figure 1 : Overview of Aesthetic Computation Tool

We associate a value 1 if there exists symmetry at specific level of VIPS tree and -1 if there isn't symmetry.

Hence The overall symmetry of page can be given as

$\mathbf{S y m}=\sum\left(\mathbf{S}_{\mathbf{i}}\right)$ where i denotes the each level of VIPS tree. 
If Sym > C then we say that symmetry is exhibited by the specific page. The constant $\mathrm{C}$ is adjusted and determined according the level of segmentation for VIPS algorithm

\subsection{Layout}

Layout $\mathrm{L}$ is a set of blocks $\mathbf{B}_{\mathbf{i}}: \mathbf{L}=\left\{\mathbf{B}_{\mathbf{1}}, \ldots, \mathbf{B}_{\mathbf{n}}\right\}$ and where each block is defined as a block $\mathrm{B}$ is a pair of two points pi: $\mathrm{B}=\left(\mathrm{p}_{1}\right.$, $\mathrm{p}_{2}$ ), the lower left and the upper right corner, a point $\mathrm{p}$ is defined by a pair of coordinates $\mathrm{x}, \mathrm{y}: \mathrm{p}=(\mathrm{x}, \mathrm{y})$.

The layout balance of a web page is checked by checking the balance of tree obtained from VIPS algorithm. The balance factor at each level is taken in to account and the overall balance is of the page is determined.

\subsection{Color Balance}

Based on various surveys relating to general human eye response to cool colors, we argue that background color and overall range of colors used within a web page should be in range of cool colors.

We also take into account the color contrast, which needs to be normally high, a high color contrast ratios indicates better reception by the web page user. Also a good contrast ratio distinguishes the background from foreground thereby increasing informative nature of a particular block on a web page.

\subsection{Font size}

VIPS algorithm uses a font size and font weight in its segmentation process, where each block extracted has uniform font size and font weight. We obtain a average font size of all blocks obtained and check if the size is in range of $12 p x$ to $18 p x$. If the average font size across all the blocks falls under the range then we infer that the font size across the web page is aesthetically enhancing the overall aesthetics of web page. The range of $12 \mathrm{px}$ to $18 \mathrm{px}$ font size is decided as optimal range as based on the most widely used web page dimensions of width $1000 \mathrm{px}+-10 \mathrm{px}$.

\subsection{Harmony and variety of web page}

Harmony being good implies good layout and proportion; else it implies a presence of variety in a web page. The proportion of web page is determined by the average ratio of block width to block height.

\subsection{Categorizing the Aesthetic Parameters} 5.6.1. Visible Parameters

Layout, Color, Font size: These parameters are implicitly visible to the users and form first impression of aesthetic appeal.

\subsubsection{Secondary computable parameters}

Economy, unity, proposition, harmony and variety: These parameters are secondary and mostly hidden to users perception at first look. But they still aid in enhancing visual aesthetic appeal.

Tests: The following table shows actually values extracted and computed using VIPS algorithm

\begin{tabular}{|c|c|c|c|c|c|c|c|c|c|}
\hline URL & Layout & Economy & Unity & $\begin{array}{l}\text { Font } \\
\text { Size }\end{array}$ & \begin{tabular}{|l|l} 
Color \\
Choice
\end{tabular} & Proportion & Harmony & Variety & Score \\
\hline aia.org/ & $\sqrt{ }$ & $\sqrt{ }$ & $\sqrt{ }$ & $\sqrt{ }$ & $\sqrt{ }$ & $X$ & $\sqrt{ }$ & $\sqrt{ }$ & 7 \\
\hline 2011mediocrity.com/ & $\sqrt{ }$ & $\mathrm{X}$ & $\mathrm{X}$ & $\sqrt{ }$ & $\sqrt{ }$ & $\sqrt{ }$ & $\mathrm{X}$ & $\sqrt{ }$ & 5 \\
\hline blastradius.com/ & $\sqrt{ }$ & $\sqrt{ }$ & $\sqrt{ }$ & $\mathrm{X}$ & $\sqrt{ }$ & $\mathrm{X}$ & $\sqrt{ }$ & $\sqrt{ }$ & 6 \\
\hline newyorker.com/ & $\sqrt{ }$ & $\sqrt{ }$ & $\sqrt{ }$ & $\mathrm{X}$ & $\sqrt{ }$ & $X$ & $\sqrt{ }$ & $\sqrt{ }$ & 6 \\
\hline lego.com/en-us/Default.aspx & $\sqrt{ }$ & $\mathrm{X}$ & $\mathrm{X}$ & $\sqrt{ }$ & $\sqrt{ }$ & $\mathrm{X}$ & $\mathrm{X}$ & $\sqrt{ }$ & 4 \\
\hline hidden-heroes.net/ & $\sqrt{ }$ & $\mathrm{X}$ & $\mathrm{X}$ & $\sqrt{ }$ & $\sqrt{ }$ & $\sqrt{ }$ & $\mathrm{X}$ & $\sqrt{ }$ & 5 \\
\hline wired.com/threatlevel/ & $\sqrt{ }$ & $\sqrt{ }$ & $\sqrt{ }$ & $\mathrm{X}$ & $\sqrt{ }$ & $\mathrm{X}$ & $\sqrt{ }$ & $\sqrt{ }$ & 6 \\
\hline marthastewart.com/ & $\sqrt{ }$ & $\sqrt{ }$ & $\sqrt{ }$ & $X$ & $\sqrt{ }$ & $\mathrm{X}$ & $\sqrt{ }$ & $\sqrt{ }$ & 6 \\
\hline whatimade.com/ & $\sqrt{ }$ & $\sqrt{ }$ & $\sqrt{ }$ & $\mathrm{X}$ & $\sqrt{ }$ & $X$ & $\sqrt{ }$ & $\sqrt{ }$ & 6 \\
\hline speakfromtheheart.com/ & $\sqrt{ }$ & $\sqrt{ }$ & $\sqrt{ }$ & $\mathrm{X}$ & $\sqrt{ }$ & $X$ & $\sqrt{ }$ & $\sqrt{ }$ & 6 \\
\hline bharatstudent.com/ & $\sqrt{ }$ & $\sqrt{ }$ & $\sqrt{ }$ & $\sqrt{ }$ & $\sqrt{ }$ & $\mathrm{X}$ & $\sqrt{ }$ & $\sqrt{ }$ & 7 \\
\hline iitb.ac.in/ & $\sqrt{ }$ & $\sqrt{ }$ & $\sqrt{ }$ & $\mathrm{X}$ & $\sqrt{ }$ & $\mathrm{X}$ & $\sqrt{ }$ & $\sqrt{ }$ & 6 \\
\hline isb.edu/isb/index.shtml & $\sqrt{ }$ & $\mathrm{X}$ & $\mathrm{X}$ & $\sqrt{ }$ & $\sqrt{ }$ & $\mathrm{X}$ & $\mathrm{X}$ & $\sqrt{ }$ & 4 \\
\hline india.gov.in/ & $\sqrt{ }$ & $\mathrm{X}$ & $\mathrm{X}$ & $\mathrm{X}$ & $\sqrt{ }$ & $X$ & $X$ & $\sqrt{ }$ & 3 \\
\hline rubyhall.com/ & $\sqrt{ }$ & $\sqrt{ }$ & $\sqrt{ }$ & $\sqrt{ }$ & $\sqrt{ }$ & $\mathrm{X}$ & $\sqrt{ }$ & $\sqrt{ }$ & 7 \\
\hline iimahd.ernet.in/ & $\sqrt{ }$ & $\sqrt{ }$ & $\sqrt{ }$ & $\sqrt{ }$ & $\sqrt{ }$ & $\mathrm{X}$ & $\sqrt{ }$ & $\sqrt{ }$ & 7 \\
\hline axisbank.com/ & $\sqrt{ }$ & $\sqrt{ }$ & $\sqrt{ }$ & $\sqrt{ }$ & $\sqrt{ }$ & $\mathrm{X}$ & $\sqrt{ }$ & $\sqrt{ }$ & 7 \\
\hline vodafone.in/pages/index.aspx & $\sqrt{ }$ & $\sqrt{ }$ & $\sqrt{ }$ & $\sqrt{ }$ & $\sqrt{ }$ & $X$ & $\sqrt{ }$ & $\sqrt{ }$ & 7 \\
\hline unipune.ac.in/ & $\sqrt{ }$ & $\mathrm{X}$ & $\mathrm{X}$ & $\mathrm{X}$ & $\sqrt{ }$ & $\mathrm{X}$ & $\mathrm{X}$ & $\sqrt{ }$ & 3 \\
\hline $\begin{array}{l}\text { maharashtra.gov.in/Pages/Hom } \\
\text { e.aspx }\end{array}$ & $\sqrt{ }$ & $\sqrt{ }$ & $\sqrt{ }$ & $\mathrm{X}$ & $\sqrt{ }$ & $\mathrm{X}$ & $\sqrt{ }$ & $\sqrt{ }$ & 6 \\
\hline bsnl.co.in/ & $\sqrt{ }$ & $\sqrt{ }$ & $\sqrt{ }$ & $\mathrm{X}$ & $\sqrt{ }$ & $\mathrm{X}$ & $\sqrt{ }$ & $\sqrt{ }$ & 6 \\
\hline solapur.nic.in/ & $\sqrt{ }$ & $X$ & $\mathrm{X}$ & $\sqrt{ }$ & $\sqrt{ }$ & $X$ & $X$ & $\sqrt{ }$ & 4 \\
\hline heromotocorp.com/ & $\sqrt{ }$ & $\sqrt{ }$ & $\sqrt{ }$ & $\sqrt{ }$ & $\sqrt{ }$ & $X$ & $\sqrt{ }$ & $\sqrt{ }$ & 7 \\
\hline mahadiscom.in/ & $\sqrt{ }$ & $\sqrt{ }$ & $\sqrt{ }$ & $\sqrt{ }$ & $\sqrt{ }$ & $X$ & $\sqrt{ }$ & $\sqrt{ }$ & 7 \\
\hline balajiwafers.com/ & $\sqrt{ }$ & $\sqrt{ }$ & $\sqrt{ }$ & $\mathrm{X}$ & $\sqrt{ }$ & $X$ & $\sqrt{ }$ & $\sqrt{ }$ & 6 \\
\hline mahadiscom.in/ & $\sqrt{ }$ & $\sqrt{ }$ & $\sqrt{ }$ & $\sqrt{ }$ & $\sqrt{ }$ & $X$ & $\sqrt{ }$ & $\sqrt{ }$ & 7 \\
\hline unipune.ac.in/ & $\sqrt{ }$ & $\sqrt{ }$ & $\sqrt{ }$ & $X$ & $\sqrt{ }$ & $X$ & $\sqrt{ }$ & $\sqrt{ }$ & 6 \\
\hline mahadiscom.in/ & $\sqrt{ }$ & $\mathrm{X}$ & $\mathrm{X}$ & $\sqrt{ }$ & $\sqrt{ }$ & $\sqrt{ }$ & $\mathrm{X}$ & $\sqrt{ }$ & 5 \\
\hline
\end{tabular}

Table 1 : Results computed for various web applications using our tool 


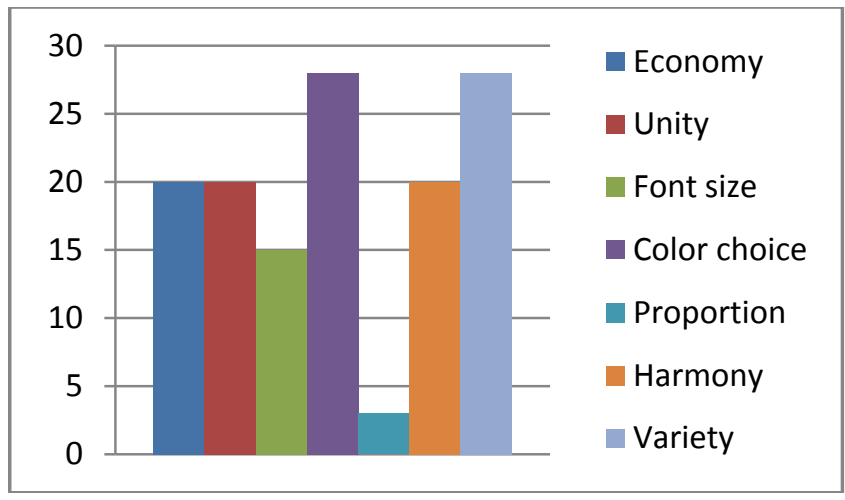

( $\mathrm{Y}$ axis indicates \# of sites qualifying the minimum threshold) Figure 2: Study of Aesthetic parameters

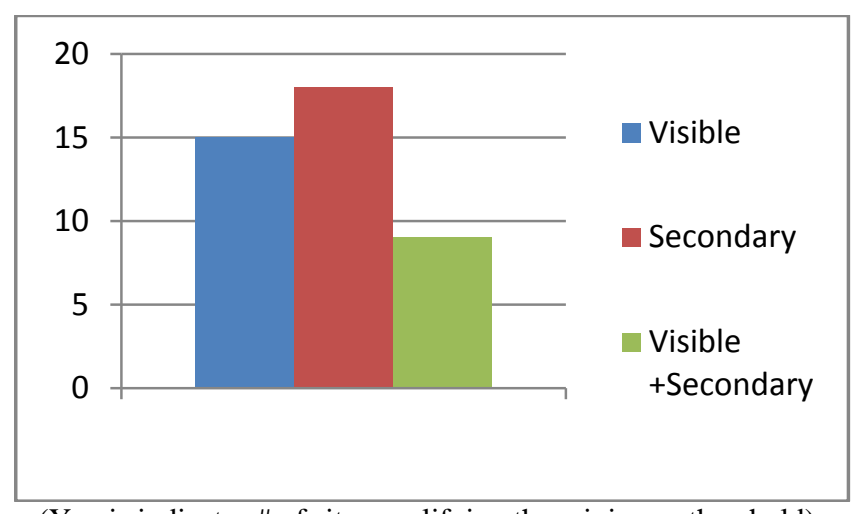

(Y axis indicates \# of sites qualifying the minimum threshold) Figure 3: Study of Aesthetic parameters Grouped

\section{SUMMARY}

With the results obtained and having an actual user's responses based on small group survey, we conclude that our attempts to quantify and measure the aesthetic features of web page are very promisable and satisfactory.

We found that font size affects the visual aspects, proportion is vital factor for determining overall visual appeal of web applications. Most site concentrated on layout and color choice which are not sufficient for overall aesthetic appeal.

Also the VIPS algorithm helps us to measure the aesthetic aspects of web page independent of the language of development of website. The VIPS algorithm is successfully able to capture the some of the aesthetic aspects of web page based on its heuristics and rules. We still find a scope to improve the correctness and accurateness of our approach by extending the VIPS algorithm with other algorithms to extract and model aesthetics present in webpage to quantify and make them measurable parameters.

\section{REFERENCES}

[1] Aesthetics, Visual Appeal, Usability and User Satisfaction: What Do the User's Eyes Tell the User's Brain? Gitte Lindgaard, Australian Journal of Emerging Technologies and Society Vol. 5, No. 1, 2007, pp: 1-14

[2] Preference ranking of screen layout principles Carolyn Salimun1, Helen C. Purchase2, David R. Simmons3, Stephen Brewster4

[3] Parizotto-Rebeiro, R., and Hammond, N. 2005. Does Aesthetics Affect the User's Perceptions of VLE's? Workshop on motivation and affect in educational software.

[4] VIPS: a Vision-based Page Segmentation Algorithm, Deng Cai ,Shipeng Yu Ji-Rong Wen ,Wei-Ying Ma ,Nov. 1, 2003 Technical Report MSR-TR-2003-79

[5] The Role of Aesthetics in Web Design, LISBETH THORLACIUS, Nordicom Review 28 (2007) 1, pp. 63-76

[6] BWAIN Artistic Web Interpretation, Linda Huber et al.

[7] Ngo, D.C., L. Teo, L.S. and Byrne, J.G. (2003), Modelling interface aesthetics, Information Sciences, 152, 25-46.

[8] Tufte, E. R. (2001). The Visual Display of Quantitative Information, Graphics Press, Cheshire

[9] Andrew Vande Moere, Nick Cawthon \& Andrea Lau The Evaluation of Aesthetic Quality in Data Visualization (http://gaugedesign.com/pdfs/visual_analytics_final.pdf)

[10] Lindgaard, G., Fernandes,G., Dudek, C. and Brown, J. 2006. Attention web designers: you have 50 milliseconds to make a good first impression. Behaviour \& Information Technology 25, 2 (Mar. 2004), 115-126.

[11] Web Site Optimization 2006. First Impressions Count in Web Design. Website optimization.

DOI=http://www.websiteoptimization.com/speed/tweak/blink/

[12] Ildus Ahmadullin; Niranjan 'amera-Venkata , "Learning Aesthetics Measure of a Document Page Layout From Designers “ „HP Laboratories HPL-2012-13. 